\title{
Editorial
}

\section{Dopamine and social anxiety disorder}

\author{
Dopamina e transtorno \\ de ansiedade social
}

Social anxiety disorder (SAnD) also known as social phobia is a common and disabling psychiatric illness. Interest in the condition has increased recently with a corresponding growth in work looking at the underlying neurobiological mechanisms of the disorder. In particular, an amygdala-based fear conditioning neurocircuitry model has been postulated with interactions between neurotransmitters such as serotonin (5HT) and dopamine (DA) attracting attention.

The clinical history of interest of DA in social anxiety is informative. Clinical observations suggested similarities between social anxiety and atypical depression (in particular the symptom of hypersensitivity to rejection) and a preferential response to monoamine oxidase inhibitors (MAOls) rather than tricyclics. As monoamine oxidase inhibitors increase synaptic levels of DA in addition to $5 \mathrm{HT}$ and noradrenaline (NA), in contrast to the tricyclics that predominantly act on $5 \mathrm{HT}$ and NA, this suggests a role for DA in SAnD. Drugs that reduce DA function, such as DA antagonists, have been reported to be associated with SAnD symptoms in patients treated for Tourette's syndrome and schizophrenia. It is not clear whether these specific subgroups are sensitive to the effects of antipsychotics or whether it is a drug class effect. Members of our group have previously reported on amphetamine misuse just before the onset of social phobia, raising the consideration that dopamine depletion may be a predisposing factor. Patients with SAnD are commonly treated with serotonergic antidepressants, however interplay with dopaminergic systems is understood to occur and the clinical effects of these agents may be mediated by processes such as D2 receptor sensitisation. ${ }^{1}$ The nature of the relationship between serotonergic and dopaminergic systems in SAnD is a topical area of recent research.

Although the use of animal models to study the role of DA in social anxiety has inherent problems because of the interactional nature of the disorder, several studies have suggested that reduced DA function is associated with increased anxiety. There is a strain of timid mice with reduced DA levels, and DA depletion in rats results in increased anxiety. The most convincing studies have been in subordinate primates (monkeys) which have reported both reduced dopamine (D2) receptor function, demonstrated by neuroendocrine studies, and neuroimaging showing reduced D2 receptor binding.

Human studies of DA and its metabolites have reported reduced cerebrospinal fluid (CSF) homovanillic acid (HVA) levels in patients with panic disorder with comorbid social anxiety compared with panic disorder patients, as well as correlations between low CSF HVA and measures of introversion. Neuroendocrine studies have not shown convincing abnormalities. The most convincing evidence for reduced DA function in humans has been shown in SPECT neuroimaging studies that reported reduced striatal DA reuptake sites $^{2}$ and, like the subordinate monkey study, reduced D2 receptor striatal binding. ${ }^{3}$ These findings are thought to reflect a reduced 
number of DA synapses as a result of reduced expression or degeneration. In this light, there may be some merit in reconceptualizing SAnD as a chronic neurodevelopmental illness.

There has also been some interesting work looking at social anxiety in DA-related disorders such as Parkinson's disease and schizophrenia. SAnD is common in Parkinson's disease and most commonly predates the motor symptoms in patients who have no prior history of anxiety. This suggests that the two conditions may be related, with a degeneration leading to reduced DA and in turn an increase in anxiety. The reduced striatal DA reuptake sites reported in the SPECT studies mentioned above resembled results found in early Parkinson's patients. Another SPECT study also found that Parkinson's patients with less DA transporters available had higher affective measures. Interestingly, anxiety symptoms in Parkinson's disease can be comorbid or related particularly to motor "off symptoms" - which respond, at least initially, to increasing dopamine with L-dopa.

The association between SAnD and schizophrenia is increasingly being recognized, with social anxiety being the most common anxiety disorder comorbid with this condition. Social anxiety often occurs prior to the onset of psychotic symptoms and before the use of antipsychotics in many cases. This again supports a neurodevelopmental aspect of SAnD. Other associations are worthy of consideration. For example, the psychological features of mercury poisoning (erythrism) such as shyness, fear of ridicule and blushing bear an uncanny resemblance to SAnD and are of interest in light of both modern community concerns of mercury exposure and increasing evidence of mercury-related DA dysfunction. ${ }^{4}$

Although work is still at an early stage, animal and human studies suggest that reduced DA function is related to social anxiety. There is also support of a neurodevelopmental basis to SAnD. Further studies addressing this area will hopefully lead to increased understanding of the role of DA and its interaction with other systems. Treatment studies using dopaminergic agents have similarly been limited though a positive outcome with olanzapine treatment has been reported ${ }^{5}$ and this again is an area that warrants further research.

Hayley M Robinson, Sean D Hood School of Psychiatry and Clinical Neurosciences, University of Western Australia, Perth, Australia

Caroline J Bell Department of Psychological Medicine, Christchurch School of Medicine and Health Science, Christchurch, New Zealand

David J Nutt Psychopharmacology Unit, University of Bristol, Bristol, United Kingdom

\section{Acknowledgements}

Hayley Robinson is grateful for the support from the Faculty of Medicine, Dentistry and Health Science at the University of Western Australia through the Jean Rogerson Scholarship.

\section{References}

1. Willner P, Hale AS, Argyropoulos S. Dopaminergic mechanism of antidepressant action in depressed patients. J Affect Disord. 2005;86(1):37-45.

2. Tiihonen J, Kuikka J, Bergstrom K, Lepola U, Koponen H, Leinonen E. Dopamine reuptake site densities in patients with social phobia. Am J Psychiatry. 1997;154(2):239-42.

3. Schneier FR, Liebowitz MR, Abi-Dargham A, Zea-Ponce Y, Lin SH, Laruelle M. Low dopamine $\mathrm{D}(2)$ receptor binding potential in social phobia. Am J Psychiatry. 2000;157(3):457-9.

4. Basu N, Klenavic K, Gamberg M, O'Brien M, Evans D, Scheuhammer $\mathrm{AM}$, Chan HM. Effects of mercury on neurochemical receptor-binding characteristics in wild mink. Environ Toxicol Chem. 2005;24(6): 1444-50.

5. Barnett SD, Kramer ML, Casat CD, Connor KM, Davidson JR. Efficacy of olanzapine in social anxiety disorder: a pilot study. J Psychopharmacol. 2002;16(4):365-8. 